\title{
Slalek
}

REPORTS SECTION

COPY

\section{TECHNIQUE FOR ESTIMATING THE MAGNITUDE AND FREQUENCY OF FLOODS IN THE HOUSTON, TEXAS, METROPOLITAN AREA}

\section{U.S. GEOLOGICAL SURVEY}

Water-Resources Investigations 80-17

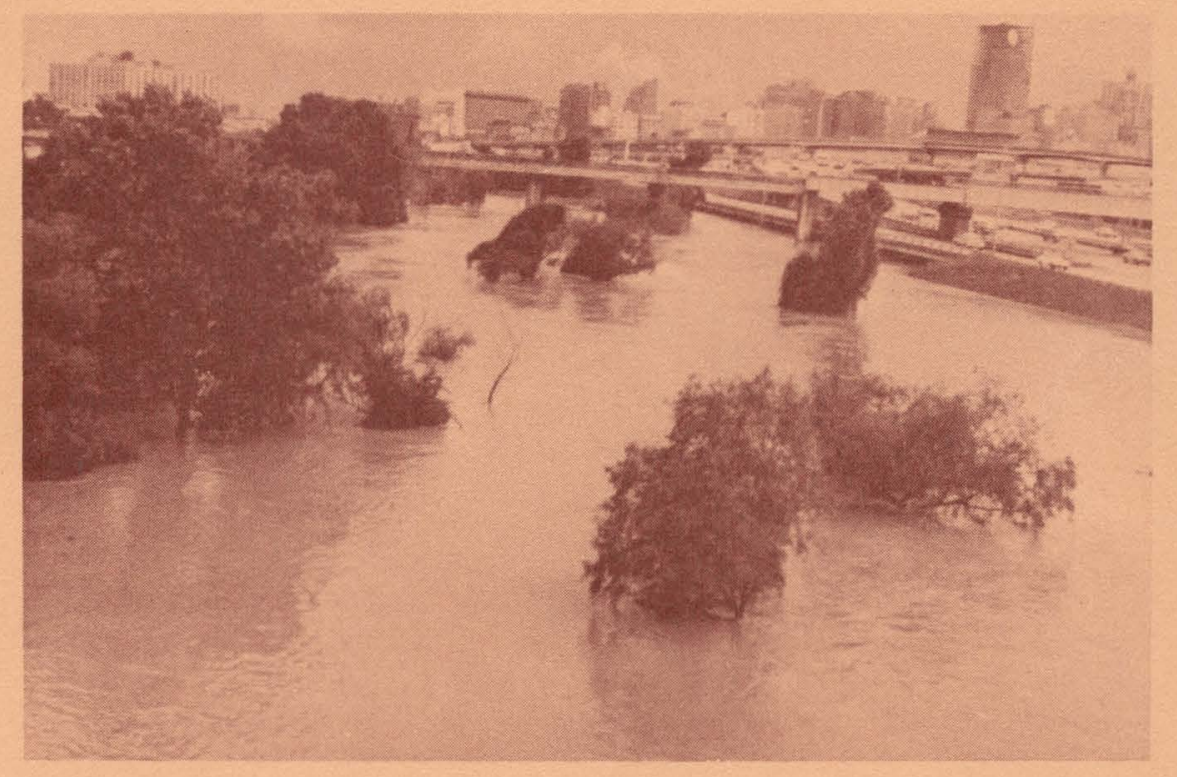

Prepared in cooperation with the City of Houston, the Harris County Flood Control District, the Texas Department of Water Resources, and the U.S. Army Corps of Engineers 


\begin{tabular}{|c|c|c|}
\hline $\begin{array}{l}\text { REPORT DOCUMENTATION } \\
\text { PAGE }\end{array}$ & 1. REPORT NO. & 3. Recipient's Accession No. \\
\hline \multirow{2}{*}{\multicolumn{2}{|c|}{$\begin{array}{l}\text { 4. Title and subtitle } \\
\text { TECHNIQUE FOR ESTIMATING THE MAGNITUDE AND FREQUENCY OF FLOODS } \\
\text { IN THE HOUSTON, TEXAS, METROPOLITAN AREA }\end{array}$}} & $\begin{array}{l}\text { 5. Report Date } \\
\text { April } 1980\end{array}$ \\
\hline & & 6. \\
\hline \multicolumn{2}{|c|}{$\begin{array}{l}\text { 7. Author(s) } \\
\text { Fred Liscum and B. C. Massey }\end{array}$} & $\begin{array}{l}\text { 8. Performing Organization Rept. No. } \\
\text { USGS/WRI }-80-17\end{array}$ \\
\hline \multicolumn{2}{|c|}{$\begin{array}{l}\text { 9. Performing Organization Name and Address } \\
\text { U.S. Geological Survey, Water Resources Division } \\
300 \text { E. Eighth Street } \\
\text { Austin, TX } 78701\end{array}$} & $\begin{array}{l}\text { 10. Project/Task/Work Unit No. } \\
\text { 11. Contract(C) or Grant(G) No. } \\
\text { (C) } \\
\text { (G) }\end{array}$ \\
\hline \multirow{2}{*}{\multicolumn{2}{|c|}{$\begin{array}{l}\text { 12. Sponsoring Organization Name and Address } \\
\text { U.S. Geological Survey, Water Resources Division } \\
300 \text { E. Eighth Street } \\
\text { Austin, TX } 78701\end{array}$}} & $\begin{array}{l}\text { 13. Type of Report \& Period Covered } \\
\text { Final }\end{array}$ \\
\hline & & 14. \\
\hline
\end{tabular}

16. Abstract (Limit: 200 words) A technique for estimating the magnitude and frequency of floods in the Houston, Texas, metropolitan area was developed by use of a multiple-regression floodfrequency analysis of flow data from unregulated streams in the area. A regression model, relating flood-peak discharge to concurrent rainfall and antecedent soil moisture conditions, was used to simulate 67-year records of annual peak discharges. Flood-frequency characteristics were determined for the simulated annual peaks and for the observed annual peaks at each of 22 gaging stations. Drainage area, bank-full channel conveyance, and percentage of urban development were used as independent variables; and weighted floodfrequency discharges were used as dependent variables in the multiple-regression analysis. Relationships applicable to unregulated streams were developed for predicting floods with recurrence intervals of $2,5,10,25,50,100$, and 500 years. Drainage basins ranged in area from 1.33 to 182 square miles. The percentage of urban development in these basins ranges from 37 to 98.9 percent.

The relationships indicate that as a basin changes from a completely natural state to one of complete urbanization, the magnitude of a 2-year peak discharge is increased by a factor of 4.2 , the magnitude of a 50 -year peak is increased by a factor of 4.9 , and the magnitude of a 100-year peak is increased by a factor of 4.9 .

17. Document Analysis a. Descriptors

*Urbanization, *Regression analysis, *Peak discharge, *Historic flood, *Flood plains, *Model studies, Estimating, Flood plain insurance

b. Identifiers/Open-Ended Terms

*Digital model, *Estimating flood frequency, *Estimating flood magnitude

c. COSATI Field/Group

18. Availability Statement

No restriction on distribution.

(See ANS1-Z39.18)

See Instructions on Reverse

\begin{tabular}{|l|l|}
$\begin{array}{l}\text { 19. Security Class (This Report) } \\
\text { UNCLASS IFIED }\end{array}$ & $\begin{array}{l}\text { 21. No. of Pages } \\
33\end{array}$ \\
\hline $\begin{array}{l}\text { 20. Security Class (This Page) } \\
\text { UNCLASSIFIED }\end{array}$ & 22. Price \\
\hline
\end{tabular}

Cover photograph, Whiteoak Bayou in flood as it approaches the downtown area, courtesy of the Harris County Flood Control District 
Abstract-1-2

Introduction--0.- 2

Purpose of this report-_. 2

Description of the area-1.- 2

Metric conversions-_-_- 4

Availability of data-_. 4

Annual peak discharges-_. 5

Observed data-1an 5

Simulated data-1..- 5

Basin characteristics-_. 8

Flood-frequency analysis-_. 15

Development of regression relationships-_an 19

Application of regression relationships-an 21

Drainage area-c.- 21

Bank-full channel conveyance-_-_.-_-_- 24

Percentage of urban development-_...- 24

Limitations and special cases-_._. 26

Selected references-_an 29 
Figure 1. Map showing the study area and locations of datacollection sites-

2. Graph showing maximum-allowable conveyance versus drainage area-

\section{TABLES}

Table 1. Period of record for gaging stations used in the floodfrequency analysis-

2. Observed annual peaks used in the analysis of observed data-

3. Summary of calibration for regression model

$$
Q_{p}=A\left(P^{b_{1}}\right)\left(D^{b_{2}}\right)\left(M^{b_{3}}\right)
$$

4. Annual maximum discharges as determined from the

simulated peaks-_... 10

5. Selected basin characteristics for sites in study-_.....- 16

6. Flood-frequency characteristics determined from observed data-

7. Flood-frequency characteristics determined from 67 years of simulated data-

8. Flood-frequency characteristics used as dependent variables-..- 20

9. Regionalized relationships for $Q_{t}$

10. Predicted flood-peak discharges for recurrence intervals of $2,5,10,25,50$, and 100 years.....................

11. Effects of urbanization on flood-frequency characteristics 


\section{TECHNIQUE FOR ESTIMATING THE MAGNITUDE}

AND FREQUENCY OF FLOODS IN THE

HOUSTON, TEXAS, METROPOLITAN AREA

By

Fred Liscum and B. C. Massey

U.S. Geological Survey

\section{ABSTRACT}

A technique for estimating the magnitude and frequency of floods in the Houston, Texas, metropolitan area was developed by use of a multiple-regression flood-frequency analysis of flow data from unregulated streams in the area. A regression model, relating flood-peak discharge to concurrent rainfall and antecedent soil moisture conditions, was used to simulate 67-year records of annual peak discharges. Flood-frequency characteristics were determined for the simulated annual peaks and for the observed annual peaks at each of 22 gaging stations. Drainage area, bank-full channel conveyance, and percentage of urban development were used as independent variables; and weighted flood-frequency discharges were used as dependent variables in the multiple regression analysis.

Relationships applicable to unregulated streams were developed for predicting floods with recurrence intervals of $2,5,10,25,50,100$, and 500 years. Drainage basins ranged in area from 1.33 to 182 square miles. The percentage of urban development in these basins ranges from 37 to 98.9 percent.

The relationships indicate that as a basin changes from a completely natural state to one of complete urbanization, the magnitude of a 2-year peak discharge is increased by a factor of 4.2 , the magnitude of a 50-year peak is increased by a factor of 4.9, and the magnitude of a 100-year peak is increased by a factor of 4.9 . 
In 1964, the U.S. Geological Survey, in cooperation with the city of Houston, began a program to define the effects of urbanization on flood characteristics in the Houston, Texas, metropolitan area. Such information is necessary for the proper design of flood-plain structures, for flood-plain management, and for the determination of flood-insurance rates.

An earlier report by Johnson and Sayre (1973) presented a technique for estimating the magnitude of flood-peak discharges for recurrence intervals of 2 to 100 years. Johnson and Sayre (1973) developed equations that related the flood-peak discharge for a particular recurrence interval to the area of the drainage basin and to the degree of urbanization.

This report was prepared in cooperation with the city of Houston, the Harris County Flood Control District, the Texas Department of Water Resources, and the U.S. Army Corps of Engineers. It presents a technique similar to that of Johnson and Sayre (1973) and should be used in preference to it. The technique should provide reliable estimates of the magnitude of floods with recurrence intervals of $2,5,10,25,50$, and 100 years for unregulated streams in the Houston metropolitan area.

The reliability of flood-frequency estimates for very large recurrence intervals is uncertain; therefore, values for the 500-year flood are omitted from this report. However, an equation is provided primarily for the use of planners who are required to compute the magnitude of a 500-year flood for special purposes such as flood-insurance studies.

In the development of this technique, the observed annual flood peaks were compiled for each of 22 gaging stations. In addition, a digital model was used to simulate a 67-year record for annual peak discharges for each of the 22 sites. Standard statistical methods recommended by the U.S. Water Resources Council (1977) were used to obtain two sets of flood-frequency discharges for each site. The two sets of values were then combined to obtain a single flood-frequency curve for each site.

Johnson and Sayre (1973) considered 12 basin characteristics to define the variation in flood-peak magnitudes in the Houston metropolitan area. Only two characteristics, size of drainage area (A), and a measure of urban development (percent impervious, I) were selected as useful. Johnson and Sayre also recognized the importance of sufficient channel capacity. The results of their report were considered in the selection of bas in characteristics for this study. Multiple-regression techniques were used to define the relationships between flood-peak magnitude and selected basin characteristics.

\section{Description of the Area}

The Houston metropolitan area (fig. 1), which encompasses about 1,000 square miles, is located on a flat coastal plain about 45 miles from the Gulf of Mexico. The soils are predominantly clays, but vary from fine sandy loams in the northern part of the area to heavier clay loams south of Buffalo Bayou. 


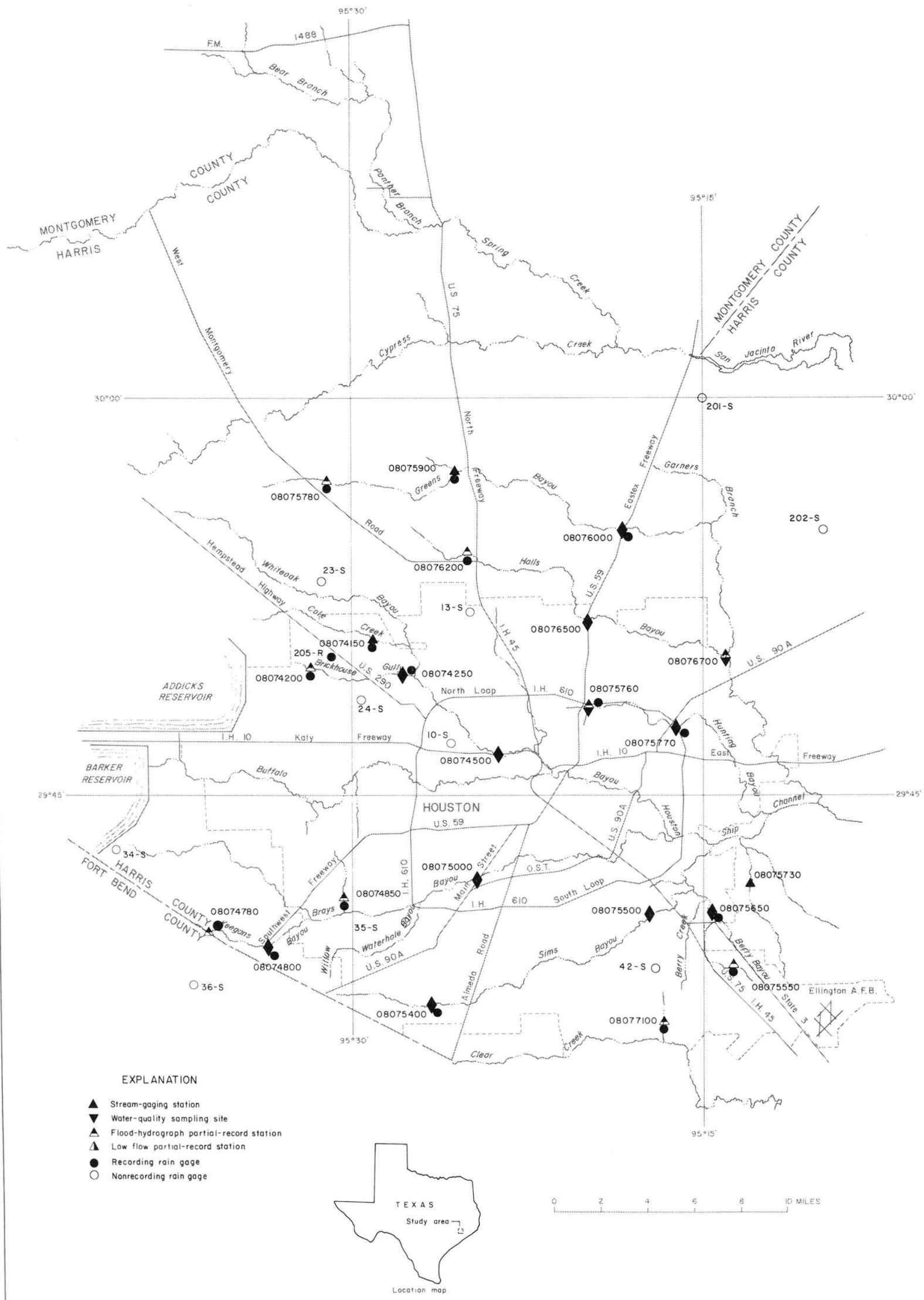

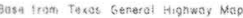


The climate is characterized by short, mild winters; long, hot summers; high relative humidity; and prevailing southeasterly winds. The mean annual temperature $(1941-70)$ is $68.9^{\circ} \mathrm{F}\left(20.5^{\circ} \mathrm{C}\right)$. The 30-year average (1941-70) rainfall for Houston is 48.19 inches, which is distributed fairly uniformly throughout the year.

The major stream draining the Houston area is Buffalo Bayou, a tributary to the San Jacinto River. Buffalo Bayou is regulated by the Barker and Addicks flood-detention reservoirs near the western limits of the area. From these reservoirs, Buffalo Bayou meanders eastward to the Houston Ship Channel, and along its course, is fed by five major tributaries: Whiteoak, Brays, Sims, Hunting, and Greens Bayous.

The channel-bed slopes ( 3 to 8 feet per mile) are relatively flat and few of the drainage-basin divides are defined accurately by natural features. Basin exchange, which is runoff to or from an adjacent basin, often results from heavy rainfall; and in many places, adjacent basins are interconnected by ditches to relieve poorly drained areas. All of the major stream channels have been improved.

Metric Conversions

For readers interested in using the metric system, the inch-pound units used in this report may be converted to metric units by the following factors:

\begin{tabular}{|c|c|c|c|c|}
\hline \multicolumn{2}{|c|}{ From } & \multirow{2}{*}{$\begin{array}{l}\text { Multiply } \\
\text { by }\end{array}$} & \multicolumn{2}{|c|}{ To obtain } \\
\hline Unit & $\begin{array}{l}\text { Abbrevi- } \\
\text { ation }\end{array}$ & & Unit & $\begin{array}{l}\text { Abbrevi- } \\
\text { ation }\end{array}$ \\
\hline $\begin{array}{l}\text { cubic foot } \\
\text { per second }\end{array}$ & $\mathrm{ft}^{3} / \mathrm{s}$ & 0.02832 & $\begin{array}{l}\text { cubic meter } \\
\text { per second }\end{array}$ & $\mathrm{m}^{3} / \mathrm{s}$ \\
\hline foot & -- & 0.3048 & meter & m \\
\hline foot squared & -- & 0.0929 & meter squared & $m^{2}$ \\
\hline foot per mile & $\mathrm{ft} / \mathrm{mi}$ & 0.189 & $\begin{array}{l}\text { meter per } \\
\text { kilometer }\end{array}$ & $\mathrm{m} / \mathrm{km}$ \\
\hline inch & -- & 25.4 & millimeter & mm \\
\hline square mile & -- & 2.590 & square kilometer & $\mathrm{km}^{2}$ \\
\hline
\end{tabular}

\section{AVAILABILITY OF DATA}

Data from 22 gaging stations were used in this analysis. Thirteen of the stations are equipped with continuous recorders; nine are equipped with flood-hydrograph recorders that record water-surface elevations only during times of storm runoff. Sixteen of these stations are instrumented to obtain a continuous rainfall record. Another 25 recording rain gages and 10 nonrecording gages are located at sites other than gaging stations. In addition, 67 years of 5-minute rainfall data (1910-76) are available from the National Weather Service station at Houston. 


\section{Annual Peak Discharges}

\section{Observed Data}

The locations of the 22 gaging stations used in this study are shown on figure 1. The period of record for each station is given in table 1 . The annual-peak discharges used in the analysis of observed data are given in table 2. Because the influence of channel capacity on the magnitude of annual peak discharges in the Houston area is greater than that of other indices of urbanization, the period of record for this analysis was selected as the period since the last major channel rectification at each of the sites.

None of the peak discharges presented in table 2 resulted from tropical storms or hurricanes. At some streamflow sites in central and south-central Texas, extremely high discharges have resulted from the intense rainfall associated with such storms. Data from these sites indicate that tropical storms may have the potential to produce flood discharges greatly in excess of those estimated when using the relationships developed in this study.

\section{Simulated Data}

A long-term record of flood peaks was simulated for each site by using a regression model developed by Johnson and Sayre (1973). The flood-frequency estimates determined from these synthetic data were combined with those determined from observed data to provide more reliable estimates of flood-peak discharges for the selected recurrence intervals.

The regression model related observed flood-peak discharges to concurrent rainfall and antecedent conditions. It has the following form:

$$
Q_{p}=a P^{b_{1}} b_{2}{ }^{b_{3}}
$$

where $Q_{p}=$ peak discharge, in cubic feet per second;

$P=$ the Theissen-weighted storm rainfall, in inches;

$D=$ storm duration, in hours, during which 85 percent of the rainfall

(P) occurred;

$M=$ the soil-moisture index as defined below;

$a=$ the regression constant; and

$b_{1}, b_{2}, b_{3}=$ regression coefficients.

The soil-moisture index, M, was defined by Johnson and Sayre (1973) as

$$
M=\left(M_{0}+P_{0}\right) k^{t}
$$

where $M=$ soil-moisture index, in inches, for the day on which the peak discharge occurred;

$M_{0}=$ the last computed soil-moisture index, in inches, for $t$ days preceding the storm; 
Table 1.-- Period of record for gaging stations used in the flood-frequency analysis

\begin{tabular}{|c|c|c|c|}
\hline Sequence & Station & $\begin{array}{l}\text { Station } \\
\text { name }\end{array}$ & $\begin{array}{l}\text { Period } \\
\text { of record }\end{array}$ \\
\hline 1 & 08074150 & Cole Creek at Deih1 Rd., Houston & $1964-76$ \\
\hline 2 & 08074200 & $\begin{array}{l}\text { Brickhouse Gully at Clarblak St., } \\
\text { Houston }\end{array}$ & $1964-76$ \\
\hline 3 & 08074250 & $\begin{array}{l}\text { Brickhouse Gully at Costa Rica St., } \\
\text { Houston }\end{array}$ & $1964-76$ \\
\hline 4 & 08074500 & Whiteoak Bayou at Houston & $1936-76$ \\
\hline 5 & 08074780 & Keegans Bayou at Keegans Rd., Houston & $\begin{array}{l}1965-71 \\
1975-76\end{array}$ \\
\hline 6 & 08074800 & Keegans Bayou at Roark Rd., Houston & $1964-76$ \\
\hline 7 & 08074850 & $\begin{array}{l}\text { Bintliff Ditch at Bissonnet St., } \\
\text { Houston }\end{array}$ & $1968-76$ \\
\hline 8 & 08075000 & Brays Bayou at Houston & $1936-76$ \\
\hline 9 & 08075400 & Sims Bayou at Hiram C1ark St., Houston & $1964-76$ \\
\hline 10 & 08075500 & Sims Bayou at Houston & $1952-76$ \\
\hline 11 & 08075550 & Berry Bayou at Gilpin St., Houston & $1965-76$ \\
\hline 12 & 08075650 & $\begin{array}{l}\text { Berry Bayou at Forest Oaks St., } \\
\text { Houston }\end{array}$ & $1964-76$ \\
\hline 13 & 08075730 & Vince Bayou at Pasadena & $1972-76$ \\
\hline 14 & 08075760 & Hunting Bayou at Falls St., Houston & $1964-76$ \\
\hline 15 & 08075770 & Hunting Bayou at $\mathrm{IH}-610$, Houston & $1964-76$ \\
\hline 16 & 08075780 & Greens Bayou at Cutten Rd., Houston & $1965-76$ \\
\hline 17 & 08075900 & $\begin{array}{l}\text { Greens Bayou at U.S. Highway } 75 \text {, } \\
\text { Houston }\end{array}$ & $1965-76$ \\
\hline 18 & 08076000 & Greens Bayou near Houston & $1952-76$ \\
\hline 19 & 08076200 & Ha11s Bayou at Deertrail St., Houston & $1965-76$ \\
\hline 20 & 08076500 & Halls Bayou at Houston & $1952-76$ \\
\hline 21 & 08076700 & Greens Bayou at Ley Rd., Houston & $1972-76$ \\
\hline 22 & 08077100 & $\begin{array}{l}\text { Clear Creek tributary at Hall Rd., } \\
\text { Houston }\end{array}$ & $1965-76$ \\
\hline
\end{tabular}


Table 2.--Observed annual peaks used in the analysis of observed data

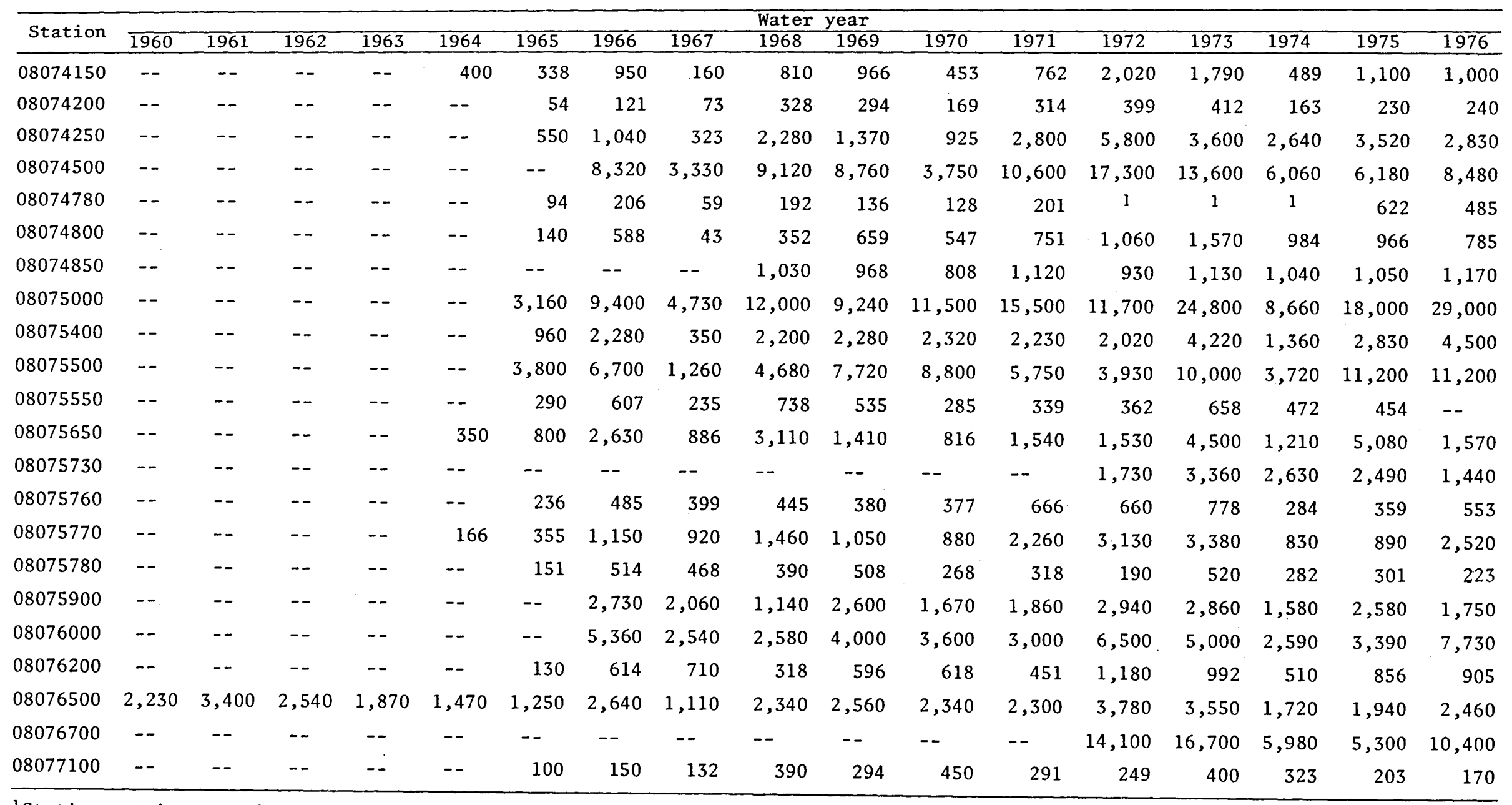

\footnotetext{
${ }^{1}$ Station not in operation.
} 
$P_{0}=$ the precipitation, in inches, on the day of the last computed soilmoisture index;

$k=$ soil-moisture depletion factor; and

$t=$ number of days between the storm and the day of the last computed soil-moisture index.

For a more detailed explanation of these variables, see Johnson and Sayre (1973).

Values for the independent variables used in the model were determined from observed streamflow and rainfall records. The period of record used in calibrating the model for each of the 22 sites was the same as that for which annual peak data were analyzed. Because the smaller peak discharges are poorly related to the weighted rainfall, only the larger peaks were used in defining the rainfall-runoff relationships.

Multiple regression techniques were used to calibrate the regression model. The calibration defined the regression constant and the parameter coefficients for each site (table 3). The standard error of estimate, Se, for the calibrated model ranges from 14.6 to 41.4 percent with a mean of 25.4 percent.

Use of the model to simulate annual-peak discharges required the use of the National Weather Service rainfall record for 1910-76 for Houston. It was assumed that this record of precipitation was representative statistically of any site within the metropolitan area, and this assumption was validated by the use of the standard statistical test of Kolmogorov-Smirnov (0stle, 1966) for goodness of fit between two data series. The test considered rainfall data over the period of calibration for both the National Weather Service gage in Houston and various representative calibration sites.

All storms that could have produced the maximum annual discharge were selected from the rainfall records. Values for rainfall amounts, storm duration, and soil-moisture index were determined for each of these storms. Because the rainfall amounts in the long-term record reflect point values, they were adjusted on the basis of drainage-area size and storm duration to reflect basin-wide averages for each site. This technique is described in U.S. Weather Bureau Technical Paper No. 40, "Rainfall Frequency Atlas of the United States" (1961, p. 6).

Peak discharges were computed for each selected storm by using the calibrated model for each site (table 3). Finally, the 67 annual maximum discharges were selected for each of the sites from the simulated peaks (table 4).

\section{Basin Characteristics}

The effects of urbanization on streamflow in the Houston metropolitan area can be attributed to two main changes within a drainage basin. First, natural soils and vegetal cover are replaced by impervious cover due to the 
Table 3.--Summary of calibration for regression model

$$
Q_{p}=a\left(P^{b_{1}}\right)\left(D^{b_{2}}\right)\left(M^{b_{3}}\right)
$$

\begin{tabular}{|c|c|c|c|c|c|c|c|}
\hline \multirow[b]{2}{*}{$\begin{array}{l}\text { Sequence } \\
\text { number }\end{array}$} & \multirow{2}{*}{$\begin{array}{l}\text { Station } \\
\text { number }\end{array}$} & \multirow[b]{2}{*}{$\begin{array}{l}\text { Constant } \\
\text { (a) }\end{array}$} & \multicolumn{3}{|c|}{ Exponent for } & \multirow{2}{*}{$\begin{array}{c}\mathrm{S}_{\mathrm{e}} \\
\text { (percent) }\end{array}$} & \multirow[b]{2}{*}{$\mathrm{MCC}^{l}$} \\
\hline & & & $\begin{array}{c}\mathrm{P} \\
\left(b_{1}\right)\end{array}$ & $\begin{array}{c}\mathrm{D} \\
\left(\mathrm{b}_{2}\right)\end{array}$ & $\begin{array}{c}M \\
\left(b_{3}\right) \\
\end{array}$ & & \\
\hline 1 & 08074150 & 244.3 & 1.382 & -0.287 & 0.080 & 16.6 & 0.961 \\
\hline 2 & 08074200 & 90.6 & .980 & -.151 & .083 & 18.8 & .912 \\
\hline 3 & 08074250 & $1,165.8$ & .987 & -.246 & .118 & 16.7 & .919 \\
\hline 4 & 08074500 & $2,306.8$ & .950 & -- & .094 & 27.0 & .857 \\
\hline 5 & 08074780 & 38.4 & 1.450 & -.145 & .246 & 25.8 & .867 \\
\hline 6 & 08074800 & 323.6 & .924 & -.245 & -- & 41.4 & .672 \\
\hline 7 & 08074850 & 490.9 & .758 & -.156 & .035 & 14.8 & .889 \\
\hline 8 & 08075000 & $5,164.2$ & 1.017 & -.197 & .091 & 17.1 & .942 \\
\hline 9 & 08075400 & 712.8 & .982 & -.121 & .087 & 26.8 & .800 \\
\hline 10 & 08075500 & $1,678.8$ & 1.220 & -.245 & .177 & 27.0 & .899 \\
\hline 11 & 08075550 & 258.2 & .717 & -.200 & .089 & 23.0 & .756 \\
\hline 12 & 08075650 & 704.7 & 1.205 & -.294 & .092 & 27.7 & .865 \\
\hline 13 & 08075730 & $1,188.5$ & .780 & -.168 & .234 & 14.6 & .902 \\
\hline 14 & 08075760 & 183.2 & .990 & -.230 & -- & 21.3 & .835 \\
\hline 15 & 08075770 & 463.4 & 1.214 & -.195 & 199 & 28.9 & .834 \\
\hline 16 & 08075780 & 98.0 & 1.160 & -.118 & .241 & 27.0 & .831 \\
\hline 17 & 08075900 & 590.2 & 1.185 & -.161 & .210 & 32.3 & .815 \\
\hline 18 & 08076000 & $1,227.4$ & 1.156 & -.240 & .117 & 26.3 & .846 \\
\hline 19 & 08076200 & 246.6 & .799 & -.102 & -- & 30.6 & .719 \\
\hline 20 & 08076500 & 839.5 & .815 & -.122 & -- & 22.3 & .832 \\
\hline 21 & 08076700 & $2,322.7$ & 1.052 & -.141 & .084 & 31.3 & .820 \\
\hline 22 & 08077100 & 128.2 & .723 & -.130 & .100 & 34.3 & .675 \\
\hline
\end{tabular}


Tahle 4.--Annual maximum discharges as determined from the simulated peaks

\begin{tabular}{|c|c|c|c|c|c|c|c|c|c|c|c|}
\hline $\begin{array}{l}\text { Water } \\
\text { year }\end{array}$ & 08074150 & 08074200 & 08074250 & 08074500 & 08074780 & $\begin{array}{l}\text { Station } \\
\text { n880748तn }\end{array}$ & 08074850 & 08075000 & 08075400 & 08075500 & 08075550 \\
\hline 1910 & 972 & 228 & 2,396 & 4,568 & 93 & 971 & 1,100 & 10,257 & 1,667 & 3,091 & 438 \\
\hline 1911 & 766 & 232 & $2,4 ! 3$ & 7,301 & 191 & 642 & 919 & 11,437 & $1, \Omega 1 \cap$ & 4,507 & 470 \\
\hline 1912 & 1,323 & 330 & 3,663 & 8,937 & 291 & 915 & 1,251 & 16,318 & 2,628 & 6,888 & 582 \\
\hline 1913 & 1,152 & 261 & 2,677 & 5,561 & 116 & 1,074 & 1,214 & 12,087 & 1,934 & 3,706 & 474 \\
\hline 1914 & 1,310 & 356 & 3,264 & 13,559 & 358 & 944 & 1,246 & 17,434 & 3,062 & 6,895 & 547 \\
\hline 1915 & 2,073 & 464 & 4,850 & 14,178 & 499 & 1,207 & 1,595 & 23,394 & 3,813 & 10,253 & 698 \\
\hline 1916 & 863 & 207 & 2,377 & 4,874 & 88 & 904 & 1,031 & 8,913 & 1,444 & 2,808 & 450 \\
\hline 1917 & 485 & 147 & 1,630 & 3,239 & 59 & 556 & 744 & 6,476 & 1,076 & 2,013 & 336 \\
\hline 1918 & 1,154 & 263 & 2,767 & 5,550 & 121 & 1,061 & 1,205 & 11,968 & 1,947 & 3,817 & 485 \\
\hline 1919 & 1,768 & 433 & 4,091 & 15,742 & 469 & 1,052 & 1,468 & 21,648 & $3,7 \cap 0$ & 8,962 & 603 \\
\hline 1920 & 921 & 268 & 2,709 & 8,893 & 233 & 682 & 1,021 & 13,188 & 2,223 & 5,201 & 454 \\
\hline 1921 & 1,171 & 282 & 3,781 & 6,082 & 201 & 909 & 1,177 & 12,625 & 1,989 & 5,568 & 642 \\
\hline 1922 & 2,227 & 488 & 5,299 & 14,271 & 555 & 1,254 & 1,652 & 24,649 & 3,983 & 11,395 & 749 \\
\hline 1323 & 978 & 275 & 2,993 & 8,319 & 249 & 797 & 1,049 & 13,603 & 2,254 & 5,705 & 496 \\
\hline 1924 & 1,678 & 375 & 4,554 & 7,446 & 312 & 1,134 & 1,440 & 17,119 & 2,765 & 7,547 & 715 \\
\hline 1925 & 581 & 167 & 1,749 & 4,661 & 67 & 645 & 821 & 7,983 & 1,333 & 2,197 & 362 \\
\hline 1926 & 1,271 & 312 & 3,244 & 8,877 & 234 & 957 & 1,231 & 15,462 & 2,521 & 5,805 & 523 \\
\hline 1927 & 327 & 113 & 1,208 & 3,313 & 60 & 462 & 609 & 5,354 & 909 & 1,796 & 259 \\
\hline 1928 & 546 & 164 & 1,795 & 3,664 & 76 & 579 & 791 & 7,496 & 1,234 & 2,452 & 356 \\
\hline 1929 & 1,996 & 441 & 4,870 & 11,911 & 442 & 1,209 & 1,569 & 22,039 & 3,521 & 9,784 & 715 \\
\hline 1.930 & 616 & 177 & 1,941 & 4,019 & 85 & 631 & 841 & 8,206 & 1,335 & 2,678 & 374 \\
\hline 1931 & 1,340 & 309 & 3,462 & 6,806 & 202 & 1,042 & 1,283 & 14,509 & 2,334 & 5,506 & 570 \\
\hline 1932 & 586 & 190 & 1,971 & 6,292 & 147 & 549 & 806 & 9,278 & 1,570 & 3,540 & 367 \\
\hline 1933 & 735 & 206 & 2,620 & 5,933 & 128 & 658 & 915 & 8,950 & 1,474 & 3,609 & 486 \\
\hline 1934 & 526 & 187 & 1,895 & 6,903 & 149 & 466 & 749 & 9,111 & 1,589 & 3,384 & 362 \\
\hline 1935 & 583 & 173 & 2,174 & 3,135 & 95 & 636 & 808 & 7,464 & 1,238 & 2,836 & 424 \\
\hline 1936 & 921 & 259 & 2,694 & 8,993 & 198 & 760 & 1,040 & 12,695 & 2,153 & 4,855 & 458 \\
\hline 1937 & 381 & 128 & 1,463 & 3,371 & 73 & 495 & 656 & 6,057 & 1,002 & 2,142 & 301 \\
\hline 1938 & 2,117 & 436 & 4,991 & 9,532 & 361 & 1,367 & 1,649 & 20,892 & 3,271 & 8,812 & 752 \\
\hline 1939 & 1,627 & 407 & 3,752 & 15,000 & 41.3 & 1,005 & 1,407 & 20,158 & 3,482 & 8,017 & 564 \\
\hline 1940 & 794 & 211 & 2,346 & 4,474 & 107 & 755 & 967 & 9,594 & 1,570 & 3,280 & 433 \\
\hline 1941 & 1,114 & 280 & 3,389 & 9,294 & 231 & 865 & 1,151 & 12,987 & 2,215 & 5,280 & 576 \\
\hline 1942 & 704 & 214 & 2,264 & 7,440 & 157 & 628 & 895 & 10,429 & 1,777 & 3,948 & 403 \\
\hline 1943 & 2,061 & 472 & 3,881 & 18,234 & 425 & 1,264 & 1,630 & 22,773 & 4,071 & 8,142 & 568 \\
\hline
\end{tabular}


Table 4.--Annual maximum discharges as determined from the simulated peaks--Continued

\begin{tabular}{|c|c|c|c|c|c|c|c|c|c|c|c|}
\hline $\begin{array}{l}\text { Water } \\
\text { year }\end{array}$ & $\begin{array}{c}08074150 \\
\text { Cont. }\end{array}$ & $\begin{array}{c}08074200 \\
\text { Cont. }\end{array}$ & $\begin{array}{c}08074250 \\
\text { Cont. }\end{array}$ & $\begin{array}{c}08074500 \\
\text { Cont. }\end{array}$ & $\begin{array}{c}08074780 \\
\text { Cont. }\end{array}$ & $\begin{array}{c}\text { Station } \\
08074800 \\
\text { Cont. }\end{array}$ & $\begin{array}{c}08074850 \\
\text { Cont. }\end{array}$ & $\begin{array}{c}08075000 \\
\text { Cont. }\end{array}$ & $\begin{array}{c}08075400 \\
\text { Cont. }\end{array}$ & $\begin{array}{c}08075500 \\
\text { Cont. }\end{array}$ & $\begin{array}{c}08075550 \\
\text { Cont. } \\
\end{array}$ \\
\hline 1944 & 2,867 & 532 & 5,122 & 14,797 & $4 n 9$ & 1,792 & 1,958 & 26,506 & 4,285 & 9,792 & 723 \\
\hline 1945 & 1,760 & 385 & 3,548 & 12,357 & 247 & 1,289 & 1,509 & 18,943 & 3,162 & 6,162 & 567 \\
\hline 1946 & 1,763 & 387 & 4,743 & 12,969 & 370 & 1,171 & 1,479 & 18,020 & 2,964 & 7,816 & 738 \\
\hline 1947 & 1,367 & 343 & 3,865 & 9,416 & 330 & 907 & 1,267 & 17,030 & 2,743 & 7,503 & 606 \\
\hline 1948 & 589 & 170 & 2,107 & 3,288 & 83 & 605 & 817 & 7,332 & 1,206 & 2,647 & 415 \\
\hline 1949 & 841 & 223 & 2,545 & 4,776 & 127 & 758 & 992 & 10,223 & 1,669 & 3,702 & 460 \\
\hline $195 n$ & 1,862 & 434 & 4,340 & 14,041 & 444 & 1,124 & 1,509 & 21,747 & 3,597 & 9,141 & 638 \\
\hline 1.951 & 742 & 193 & 2,331 & 4,416 & 86 & 757 & 937 & 8,356 & 1,355 & 2,867 & 446 \\
\hline 1952 & 536 & 181 & 1,885 & 6,233 & 123 & 488 & 763 & 8,811 & 1,505 & 3,083 & 367 \\
\hline 1953 & 665 & 200 & 2,320 & 5,212 & 140 & 639 & 861 & 9,642 & 1,558 & 3,764 & 422 \\
\hline 1954 & 773 & 219 & 1,965 & 7,716 & 111 & 739 & 961 & 10,523 & 1,843 & 2,980 & 370 \\
\hline 1955 & 674 & 195 & 2,486 & 3,533 & 120 & 616 & 871 & 8,442 & 1,393 & 3,400 & 468 \\
\hline 1956 & 419 & 137 & 1,629 & 2,721 & 65 & 465 & 677 & 5,955 & 995 & 2,039 & 339 \\
\hline 1957 & 953 & 241 & 2,769 & 5,278 & 134 & 843 & 1,071 & 10,744 & 1,766 & 3,890 & 495 \\
\hline 1958 & 925 & 247 & 2,960 & 8,410 & 175 & 805 & 1,041 & 11,693 & 2,031 & 4,654 & 516 \\
\hline 1959 & $92 ?$ & 239 & 2,716 & 8,010 & 166 & 839 & 1,043 & 11,470 & 1,917 & 4,157 & 482 \\
\hline 1960 & 2,370 & 501 & 5,456 & 18,771 & 577 & 1,491 & 1,779 & 24,856 & 4,296 & 11,726 & 769 \\
\hline 1961 & 796 & 239 & 2,347 & 8,495 & 173 & 700 & 954 & 11,681 & 2,016 & 4,058 & 434 \\
\hline 1962 & 1,000 & 263 & 2,919 & 8,417 & 178 & 829 & 1,087 & 12,805 & 2,159 & 4,581 & 505 \\
\hline 1963 & 847 & 216 & 2,408 & 5,534 & 134 & 818 & 1,012 & 10,183 & 1,667 & 3,702 & 446 \\
\hline 1964 & 376 & 130 & 1,470 & 3,218 & 65 & 418 & 634 & 6,123 & 1,007 & 2,058 & 305 \\
\hline 1965 & 865 & 224 & 2,526 & $6, \cap 82$ & 120 & 795 & 1,012 & 10,261 & 1,668 & 3,556 & 457 \\
\hline 1966 & 807 & 222 & 2,635 & 6,463 & 150 & 701 & 962 & 10,655 & 1,774 & 3,926 & 474 \\
\hline 1967 & 661 & 173 & 1,828 & 3,250 & 60 & 756 & 900 & 7,444 & 1,238 & 2,113 & 366 \\
\hline 1968 & 1,572 & 376 & 4,004 & 11,007 & 355 & 1,021 & 1,371 & 18,759 & 3,061 & 7,933 & 610 \\
\hline 1969 & 674 & 194 & 2,215 & 4,465 & 112 & 631 & 874 & 9,086 & 1,472 & 3,265 & 413 \\
\hline 1970 & 499 & 165 & 1,660 & 5,141 & 97 & 531 & 736 & 7,966 & 1,349 & 2,647 & 319 \\
\hline 1971 & 977 & 249 & 2,922 & 6,298 & 152 & 831 & 1,080 & 11,154 & $.1,830$ & 4,234 & 516 \\
\hline 1972 & 813 & 225 & 2,467 & 5,805 & 144 & 702 & 965 & 10,902 & 1,753 & 3,996 & 438 \\
\hline 1973 & 1,866 & 468 & 4,204 & 18,572 & 566 & 1,040 & 1,510 & 22,883 & 4,069 & 9,672 & 607 \\
\hline 1974 & 643 & 181 & 2,105 & 5,501 & 107 & 657 & 864 & 7,861 & 1,352 & 2,746 & 410 \\
\hline 1975 & 496 & 171 & 1,654 & 5,948 & 112 & 463 & 732 & 8,289 & 1,426 & 2,829 & 346 \\
\hline 1976 & 2,207 & 434 & 4,517 & 10,503 & 304 & 1,513 & 1,696 & 21,171 & 3,356 & 7,841 & 677 \\
\hline
\end{tabular}


Table 4.--Annual maximum discharges as determined from the simulated peaks--Continued

\begin{tabular}{|c|c|c|c|c|c|c|c|c|c|c|c|}
\hline Water & & & & & & Station & & & & & \\
\hline year & 08075650 & 08075730 & 08075760 & 08075770 & 08075780 & 08075900 & 08076000 & 08076200 & 08076500 & 08076700 & $080771 n$ n \\
\hline 1910 & 2,101 & 1,237 & 627 & 935 & 166 & 1,026 & 2,619 & 674 & 2,191 & 4,671 & 217 \\
\hline 1911 & 1,766 & 2,410 & 399 & 1,491 & 359 & 1,907 & 2,993 & 539 & 1,747 & 5,788 & 251 \\
\hline 1912 & 2,875 & 3,109 & 597 & 2,219 & 494 & 2,739 & 4,552 & 718 & 2,363 & 7,924 & 325 \\
\hline 1913 & 2,405 & 1,738 & 705 & 1,104 & 211 & 1,241 & 3,117 & 755 & 2,479 & 5,634 & 238 \\
\hline 1914 & 2,618 & 2,769 & 615 & 2,323 & 570 & 3,069 & 4,640 & 829 & 2,682 & 9,999 & 325 \\
\hline 1915 & 4,116 & 3,819 & 814 & 3,337 & 754 & 4,151 & 6,701 & 983 & 3,223 & 12,199 & 410 \\
\hline 1916 & 1,996 & 1,327 & 574 & 871 & 172 & 1,008 & 2,312 & 575 & 1,867 & 4,180 & 211 \\
\hline 1917 & 1,193 & 1,174 & 347 & 639 & 126 & 721 & 1,593 & 413 & 1,334 & 2,835 & 168 \\
\hline 1918 & 2,450 & 1,839 & 691 & 1,161 & 247 & 1,300 & 3,130 & 736 & 2,415 & 5,505 & 244 \\
\hline 1919 & 3,411 & 3,285 & 721 & 2,973 & 708 & 3,873 & 5,962 & 968 & 3,158 & 12,285 & 378 \\
\hline 1920 & 2,006 & 2,502 & 446 & 1,714 & 413 & 2,225 & 3,477 & 624 & 2,024 & 6,966 & 273 \\
\hline 1921 & 2,865 & 3,305 & 573 & 1,865 & 379 & 2,033 & 3,633 & 567 & 1,852 & 5,124 & 310 \\
\hline 1922 & 4,475 & 4,328 & 841 & 3,708 & 838 & 4,599 & 7,232 & 988 & 3,255 & 12,613 & 434 \\
\hline 1923 & 2,192 & 2,888 & 511 & 1,883 & 448 & 2,390 & 3,692 & 608 & 1,980 & 6,827 & 288 \\
\hline 1924 & 3,715 & 3,631 & 743 & 2,535 & 524 & 2,862 & 4,970 & 759 & 2,483 & 7,530 & 367 \\
\hline 1925 & 1,301 & 1,410 & 410 & 656 & 130 & 790 & 1,891 & 547 & 1,785 & 4,016 & 171 \\
\hline 1926 & 2,665 & 2,345 & 618 & 1,835 & 392 & 2,248 & 4,153 & 762 & 2,502 & 7,755 & 295 \\
\hline 1927 & 801 & 1,321 & 286 & 585 & 140 & 748 & 1,264 & 366 & 1,188 & 2,647 & 146 \\
\hline 1928 & 1,315 & 1,352 & 364 & 767 & 156 & 897 & 1,878 & 458 & 1,501 & 3,372 & 184 \\
\hline 1929 & 4,096 & 3,835 & 806 & 3,146 & 688 & 3,847 & 6,384 & 919 & 3,035 & 10,982 & 401 \\
\hline 1930 & 1,459 & 1,414 & 396 & 841 & 170 & 974 & 2,061 & 481 & 1,558 & 3,723 & 193 \\
\hline 1931 & 2,900 & 2,348 & 677 & 1,738 & 345 & 1,987 & 3,991 & 738 & 2,415 & 6,661 & 296 \\
\hline 1932 & 1,385 & 2,085 & 347 & 1,176 & 293 & 1,546 & 2,355 & 467 & 1,521 & 4,728 & 216 \\
\hline 1933 & 1,860 & 2,417 & 412 & 1,233 & 260 & 1,371 & 2,430 & 539 & 1,732 & 4,444 & 242 \\
\hline 1934 & 1,250 & 1,987 & 289 & 1,144 & 295 & 1,523 & 2,264 & 456 & 1,464 & 4,879 & 211 \\
\hline 1935 & 1,508 & 1,989 & 400 & 963 & 202 & 1,071 & 1,968 & 461 & 1,492 & 3,083 & 210 \\
\hline 1936 & 2,016 & 2,347 & 497 & 1,567 & 358 & 1,966 & 3,313 & 701 & 2,264 & 6,993 & 262 \\
\hline 1937 & 970 & 1,552 & 306 & 703 & 165 & 871 & 1,488 & 374 & 1,216 & 2,782 & 165 \\
\hline 1938 & 4,380 & 3,440 & 914 & 2,806 & 563 & 3,196 & 6,056 & 931 & 3,052 & 9,618 & 392 \\
\hline 1939 & 3,130 & 2,922 & 692 & 2,654 & 628 & 3,418 & 5,460 & 945 & 3,062 & 11,539 & 355 \\
\hline 1940 & 1,836 & 1,634 & 480 & 1,038 & 205 & 1,187 & 2,491 & 549 & 1,790 & 4,306 & 221 \\
\hline 1941 & 2,597 & 2,854 & 556 & 1,758 & 407 & 2,175 & 3,520 & 627 & 2,022 & 7,085 & 296 \\
\hline 1942 & 1,619 & 2,091 & 414 & 1,284 & 300 & 1,626 & 2,703 & 609 & 1,966 & 5,645 & 232 \\
\hline 1943 & 3,606 & 2,342 & 900 & 2,651 & 592 & 3,367 & 6,064 & 1,222 & 3,942 & 13,793 & 364 \\
\hline
\end{tabular}


Table 4.--Annual maximum discharges as determined from the simulated peaks--Continued

\begin{tabular}{|c|c|c|c|c|c|c|c|c|c|c|c|}
\hline $\begin{array}{l}\text { Water } \\
\text { year }\end{array}$ & $\begin{array}{c}08075650 \\
\text { Cont. }\end{array}$ & $\begin{array}{c}08075730 \\
\text { Cont. }\end{array}$ & $\begin{array}{c}08075760 \\
\text { Cont. }\end{array}$ & $\begin{array}{c}08075770 \\
\text { Cont. }\end{array}$ & $\begin{array}{c}08075780 \\
\text { Cont. }\end{array}$ & $\begin{array}{c}\text { Station } \\
08075900 \\
\text { Cont. }\end{array}$ & $\begin{array}{c}08076000 \\
\text { Cont. }\end{array}$ & $\begin{array}{c}08076200 \\
\text { Cont. }\end{array}$ & $\begin{array}{c}08076500 \\
\text { Cont. }\end{array}$ & $\begin{array}{c}08076700 \\
\text { Cont. }\end{array}$ & $\begin{array}{c}08077100 \\
\text { Cont. }\end{array}$ \\
\hline 1944 & 5,156 & 2,568 & 1,233 & 2,942 & 562 & 3,472 & 7,488 & 1,346 & 4,460 & 14,091 & 404 \\
\hline 1945 & 3,210 & 2,397 & 888 & 1,928 & 394 & 2,289 & 4,961 & 1,101 & 3,600 & 10,646 & 309 \\
\hline 1946 & 3,897 & 3,777 & 769 & 2,639 & 610 & 3,267 & 5,119 & 820 & 2,653 & 9,576 & 378 \\
\hline 1947 & 2,987 & 3,499 & 593 & 2,451 & 560 & 3,047 & 4,804 & 716 & 2,355 & 8,357 & 342 \\
\hline 1948 & 1,506 & 1,747 & 374 & 878 & 176 & 964 & 1,914 & 413 & 1,328 & 3,023 & 202 \\
\hline 1949 & 1,959 & 2,310 & 482 & 1,188 & 241 & 1,369 & 2,701 & 551 & 1,795 & 4,568 & 237 \\
\hline 1950 & 3,666 & 3,365 & 762 & 2,964 & 677 & 3,760 & 6,099 & 959 & 3,138 & 11,727 & 382 \\
\hline 1951 & 1,807 & 1,601 & 474 & 919 & 172 & 985 & 2,191 & 493 & 1,596 & 3,468 & 212 \\
\hline 1952 & 1,265 & 1,851 & 311 & 1,000 & 243 & 1,310 & 2,177 & 472 & 1,519 & 4,659 & 200 \\
\hline 1953 & 1,618 & 2,243 & 409 & 1,227 & 279 & 1,495 & 2,534 & 538 & 1,761 & 4,503 & 229 \\
\hline 1954 & 1,595 & 1,182 & 487 & 916 & 192 & 1,147 & 2,507 & 703 & 2,269 & 6,018 & 200 \\
\hline 1955 & 1,731 & 2,371 & 384 & 1,166 & 248 & 1,301 & 2,279 & 426 & 1,367 & 3,484 & 233 \\
\hline 1956 & 1,096 & 1,471 & 286 & 683 & 145 & 779 & 1,486 & 349 & 1,115 & 2,527 & 171 \\
\hline 1957 & 2,192 & 1,974 & 541 & 1,265 & 253 & 1,418 & 2,866 & 588 & 1,912 & 4,724 & 250 \\
\hline 1958 & 2,194 & 2,609 & 541 & 1,540 & 331 & 1,801 & 3,132 & 761 & 2,463 & 6,683 & 270 \\
\hline 1959 & 2,118 & 2,025 & 538 & 1,337 & 304 & 1,681 & 2,962 & 669 & 2,154 & 6,086 & 249 \\
\hline 1960 & 4,538 & 4,662 & 1,071 & 3,861 & 880 & 4,790 & 7,318 & 1,396 & 4,520 & 14,795 & 442 \\
\hline 1961 & 1,771 & 1,801 & 442 & 1,315 & 308 & 1,695 & 2,936 & 626 & 2,021 & 6,400 & 237 \\
\hline 1962 & 2,279 & 2,240 & 531 & 1,463 & 325 & 1,809 & 3,263 & 716 & 2,328 & 6,897 & 263 \\
\hline 1963 & 1,939 & 1,909 & 524 & 1,180 & 256 & 1,433 & 2,641 & 573 & 1,863 & 4,852 & 227 \\
\hline 1964 & 968 & 1,376 & 256 & 659 & 145 & 796 & 1,498 & 344 & 1,114 & 2,754 & 162 \\
\hline 1965 & 1,990 & 1,771 & 506 & 1,139 & 225 & 1,284 & 2,678 & 570 & 1,852 & 4,590 & 233 \\
\hline 1966 & 1,938 & 2,261 & 442 & 1,301 & 282 & 1,564 & 2,751 & 548 & 1,784 & 5,350 & 246 \\
\hline 1967 & 1,508 & 999 & 483 & 654 & 117 & 708 & 1,829 & 532 & 1,711 & 3,296 & 178 \\
\hline 1968 & 3,264 & 3,228 & 675 & 2,556 & 572 & 3,173 & 5,266 & 827 & 2,715 & 9,559 & 351 \\
\hline 1969 & 1,617 & 1,837 & 396 & 1,051 & 222 & 1,232 & 2,349 & 481 & 1,558 & 4,090 & 216 \\
\hline 1970 & 1,165 & 1,439 & 339 & 849 & 195 & 1,076 & 1,945 & 489 & 1,577 & 4,040 & 184 \\
\hline 1971 & 2,270 & 2,238 & 532 & 1,396 & 282 & 1,574 & 3,015 & 583 & 1,888 & 4,945 & 262 \\
\hline 1972 & 1,862 & 1,985 & 447 & 1,258 & 271 & 1,529 & 2,854 & 557 & 1,816 & 5,184 & 238 \\
\hline 1973 & 3,499 & 3,517 & 730 & 3,320 & 829 & 4,355 & 6,308 & 1,028 & 3,305 & 13,485 & 399 \\
\hline 1974 & 1,572 & 1,574 & 414 & 892 & 217 & 1,170 & 1,979 & 461 & 1,478 & 4,150 & 203 \\
\hline 1975 & 1,141 & 1,658 & 297 & 917 & 222 & 1,202 & 2,024 & 458 & 1,469 & 4,399 & 190 \\
\hline 1976 & 4,282 & 2,476 & 1,016 & 2,386 & 453 & 2,735 & 5,929 & 1,075 & 3,537 & 10,446 & 360 \\
\hline
\end{tabular}


construction of roads, buildings, plants, shopping centers, and parking lots. Second, the natural condition of the channel is altered by channel-improvements, which may mean that the channel is cleaned of vegetation, the channel is concrete lined, or the channel is replaced by a storm-sewer system. A11 methods of improvement provide a more hydraulically efficient cross-sectional shape. Basin characteristics selected for this study attempted to quantify these changes in a basin.

The basin characteristics used are (1) drainage area, (2) bank-full channel conveyance, and (3) percentage of urban development. Drainage area, A, in square miles, is defined as the total contributing drainage area at the gaging-station location. It is determined by planimetering the delineated area on topographic maps.

Bank-full channel conveyance, $K$, is defined as the value computed for conveyance at the controlling section, when the stage elevation is equal to that of the lower bank, using Manning's equation for open-channel flow:

$$
K=\frac{1.49}{n} A_{x} R^{\frac{2}{3}}
$$

where $A_{X}=$ bank-full cross-sectional area, in feet squared;

$R=$ bank-ful 1 hydraulic radius, in feet; and

$n=$ Manning's roughness coefficient.

This measure of conveyance is an indication not only of channel capacity but also of the relative efficiency of a channel. $A_{x}$ and $R$ are determined by field surveys at the controlling section of a channel for the gaging station. Manning's $n$ is selected in the field by experienced personnel.

Johnson and Sayre (1973, p. 44) noted:

"The estimated T-year discharges from the relationship are design values. Sufficient channel capacity must be provided or inundation, resulting from temporary ponding, will occur in parts of the basin. Unless adequate channel capacity is provided, flooding can occur at low points along the channel as a result of channel flooding or at street intersections and grade separations that cannot be adequately drained.

"Because the discharges are design values, they should not be used to predict water-surface elevations along a channel that has a capacity less than the selected T-year discharge indicated by the relation."

These statements emphasize the importance of channel capacity in determining flood-peak magnitude for this coastal area.

Johnson and Sayre (1973, p. 5) also noted the increase in the magnitude of flood peaks following channel improvements. Espey and Winslow (1968, p. 55) discussed the effects of changes in channel conveyance on the time of rise and on the unit-peak discharge of streams in the Houston area. Changes in the magnitude of peak discharges in the Houston area can be largely attributed to channel improvements. This study has shown channel conveyance to be a more important parameter in estimating the magnitude and frequency of floods in Houston than other commonly used indices of urbanization. 
The percentage of urban development, $A_{D}$, is defined as the percentage of the total contributing drainage area within 200 feet of streets, roads, parking lots, and industrial sites that is drained by open street ditches or storm sewers. $A D$ is highly correlated with the percentage of impervious area in a basin, I, as used by Johnson and Sayre (1973), but is much easier to determine accurately from aerial photographs. table 5 .

The values for $A, K$, and $A D$, as determined for this study, are given in

\section{FLOOD-FREQUENCY ANALYSIS}

The flood-frequency characteristics as computed from the observed annualpeak discharge data are given in table 6; the observed station skew was applied to the values because of a lack of information concerning skew variation in an urbanizing coastal area. Low outliers that caused an abnormal negative bias to the annual-peak discharge data were discounted according to the Water Resources Council (1977) guidelines.

The flood-frequency characteristics computed from the simulated annualpeak discharge data are given in table 7. The skew value determined from the 67-year series of simulated discharges at each station was used in computing these characteristics.

The determination of regional relationships for predicting flood-frequency characteristics requires one set of flood-frequency values (dependent variables) for each of the sites in the study area. Water Resources Council (1977) guidelines suggest that flood-frequency curves determined from observed data may be adjusted by the use of simulated flood-frequency values, but the guidelines require that any adjustments incorporate the relative accuracy of the simulated and observed data.

Several methods of adjusting or weighting the flood-frequency curves were evaluated. These methods were (1) averaging, (2) weighting based on length of observed record, and (3) weighting based on error characteristics.

In the averaging method, the weighted values are obtained by averaging the results from the observed and simulated data for each recurrence interval. This procedure is based on the assumption that each data series represents conditions that are equally likely to occur at a site under the stated degree of urbanization.

The weighting method based on the length of observed record requires a specified amount of observed data to define a flood peak for a particular recurrence interval. If this amount of observed data is available, then the weighted flood-frequency value is equal to the observed value. Otherwise, the weighted flood-frequency value is equal to some combination of the observed value and the simulated value. In this method, the observed data are weighted more heavily than the simulated data.

In the method of weighting based on error characteristics, the weighted values are determined by considering the relative errors present in each data series. The procedure for computing relative error is analogous to a variance 
Table 5.--Selected basin characteristics for sites in study

\begin{tabular}{|c|c|c|c|}
\hline Station & A & K & $\begin{array}{c}A_{D} \\
\text { (percent) }\end{array}$ \\
\hline 08074150 & 8.81 & $1.7 \times 10^{5}$ & 54.0 \\
\hline 08074200 & 2.56 & $2.1 \times 10^{4}$ & 54.7 \\
\hline 08074250 & 11.4 & $2.3 \times 10^{5}$ & 77.5 \\
\hline 08074500 & 84.7 & $1.7 \times 10^{6}$ & 57.7 \\
\hline 08074780 & 7.87 & $3.5 \times 10^{4}$ & 44.9 \\
\hline 08074800 & 12.0 & $5.6 \times 10^{4}$ & 55.7 \\
\hline 08074850 & 4.29 & $8.2 \times 10^{4}$ & 88.3 \\
\hline 08075000 & 88.4 & $2.8 \times 10^{6}$ & 64.4 \\
\hline 08075400 & 20.2 & $2.8 \times 10^{5}$ & 69.3 \\
\hline 08075500 & 64.0 & $5.3 \times 10^{5}$ & 73.7 \\
\hline 08075550 & 2.87 & $3.6 \times 10^{4}$ & 71.8 \\
\hline 08075650 & 10.1 & $4.5 \times 10^{5}$ & 85.3 \\
\hline 08075730 & 8.21 & $2.0 \times 10^{5}$ & 89.4 \\
\hline 08075760 & 2.75 & $5.1 \times 10^{4}$ & 98.9 \\
\hline 08075770 & 14.7 & $2.7 \times 10^{5}$ & 95.0 \\
\hline 08075780 & 8.73 & $1.2 \times 10^{4}$ & 47.2 \\
\hline 08075900 & 36.1 & $8.9 \times 10^{4}$ & 37.0 \\
\hline 08076000 & 69.6 & $2.9 \times 10^{5}$ & 43.9 \\
\hline 08076200 & 8.69 & $3.1 \times 10^{4}$ & 52.8 \\
\hline 08076500 & 28.3 & $1.0 \times 10^{5}$ & 74.1 \\
\hline 08076700 & 182.0 & $9.3 \times 10^{5}$ & 60.6 \\
\hline 08077100 & 1.33 & $2.0 \times 10^{4}$ & 93.2 \\
\hline
\end{tabular}


Table 6.--Flood-frequency characteristics determined from observed data

\begin{tabular}{|c|c|c|c|c|c|c|c|c|c|}
\hline \multirow[b]{2}{*}{ Station } & \multirow[b]{2}{*}{$\mathrm{Q}_{2}$} & \multirow[b]{2}{*}{$Q_{5}$} & \multirow[b]{2}{*}{$Q_{10}$} & \multirow[b]{2}{*}{$Q_{25}$} & \multirow[b]{2}{*}{$\mathrm{Q}_{50}$} & \multirow[b]{2}{*}{$Q_{100}$} & \multicolumn{2}{|c|}{ Annual peak data } & \multirow[b]{2}{*}{ Skew } \\
\hline & & & & & & & $\begin{array}{l}\text { Mean } \\
\text { of logs }\end{array}$ & $\begin{array}{l}\text { Standard } \\
\text { deviation }\end{array}$ & \\
\hline 08074150 & 750 & 1,290 & 1,650 & 2,110 & 2,430 & 2,750 & 2.8490 & 0.304 & -0.520 \\
\hline 08074200 & 220 & 345 & 420 & 500 & 545 & 590 & 2.3000 & .284 & -.890 \\
\hline 08074250 & 1,930 & 3,640 & 4,820 & 6,260 & 7,260 & 8,210 & 3.2430 & .372 & -.700 \\
\hline 08074500 & 7,920 & 12,090 & 14,880 & 18,400 & 21,000 & 23,600 & 3.8890 & .227 & -.270 \\
\hline 08074780 & 175 & 335 & 485 & 735 & .970 & 1,250 & 2.2630 & .323 & .370 \\
\hline 08074800 & 700 & 1,020 & 1,230 & 1,490 & 1,690 & 1,880 & 2.8380 & .200 & -.190 \\
\hline 08074850 & 1,040 & 1,130 & 1,160 & 1,190 & 1,210 & 1,220 & 3.0090 & .050 & -1.02 \\
\hline 08075000 & 12,520 & 19,230 & 24,030 & 30,430 & 35,420 & 40,600 & 4.0960 & .222 & -.030 \\
\hline 08075400 & 2,210 & 3,200 & 3,830 & 4,600 & 5,150 & 5,680 & 3.3340 & .200 & -.310 \\
\hline 08075500 & 4,350 & 7,220 & 9,270 & 11,980 & 14,040 & 16,140 & 3.6260 & .273 & -.260 \\
\hline 08075550 & 425 & 585 & 685 & 815 & 910 & 1,000 & 2.6280 & .164 & -.060 \\
\hline 08075650 & 1,550 & 2,890 & 3,960 & 5,540 & 6,850 & 8,280 & 3.1850 & .325 & -.100 \\
\hline 08075730 & 2,260 & 2,980 & 3,410 & 3,920 & 4,280 & 4,620 & 3.3480 & .148 & -.250 \\
\hline 08075760 & 445 & 600 & 695 & 820 & 910 & 995 & 2.6460 & .155 & -.070 \\
\hline 08075770 & 1,240 & 2,300 & 3,000 & 3,820 & 4,390 & 4,900 & 3.0440 & .370 & -.780 \\
\hline 08075780 & 330 & 455 & 535 & 625 & 685 & 745 & 2.5050 & .179 & -.360 \\
\hline 08075900 & 2,140 & 2,690 & 2,990 & 3,300 & 3,500 & 3,670 & 3.3180 & .132 & -.600 \\
\hline 08076000 & 3,350 & 5,010 & 6,210 & 7,810 & 9,080 & 10,400 & 3.5270 & .206 & .070 \\
\hline 08076200 & 650 & 890 & 1,040 & 1,210 & 1,330 & 1,440 & 2.8010 & .174 & -.360 \\
\hline 08076500 & 2,050 & 2,720 & 3,130 & 3,620 & 3,970 & 4,310 & 3.3070 & .150 & -.190 \\
\hline .08076700 & 9,650 & 14,650 & 18,060 & 22,430 & 25,710 & 29,010 & 3.9780 & .221 & -.180 \\
\hline 08077100 & 245 & 360 & 430 & 515 & 570 & 625 & 2.3770 & .209 & -.420 \\
\hline
\end{tabular}


Table 7.--Flood-frequency characteristics determined from 67 years of simulated data

\begin{tabular}{|c|c|c|c|c|c|c|c|c|c|}
\hline \multirow[b]{2}{*}{ Station } & \multirow[b]{2}{*}{$\mathrm{Q}_{2}$} & \multirow[b]{2}{*}{$\mathrm{Q}_{5}$} & \multirow[b]{2}{*}{$Q_{10}$} & \multirow[b]{2}{*}{$Q_{25}$} & \multirow[b]{2}{*}{$Q_{50}$} & \multirow[b]{2}{*}{$Q_{100}$} & \multicolumn{2}{|c|}{$\begin{array}{c}\text { Simulated annual } \\
\text { peak data } \\
\end{array}$} & \multirow[b]{2}{*}{ Skew } \\
\hline & & & & & & & $\begin{array}{l}\text { Mean } \\
\text { of } \operatorname{logs} \\
\end{array}$ & $\begin{array}{l}\text { Standard } \\
\text { deviation }\end{array}$ & \\
\hline 08074150 & 935 & 1,450 & 1,840 & 2,390 & 2,840 & 3,320 & 2.9760 & 0.223 & 0.160 \\
\hline 08074200 & 250 & 350 & 420 & 515 & 585 & 665 & 2.4060 & .167 & .220 \\
\hline 08074250 & 2,720 & 3,700 & 4,360 & 5,210 & 5,840 & 6,500 & 3.4390 & .158 & .080 \\
\hline 08074500 & 6,880 & 10,600 & 13,400 & 17,300 & 20,530 & 23,960 & 3.8440 & .218 & .180 \\
\hline 08074780 & 165 & 290 & 400 & 560 & 705 & 870 & 2.2320 & .282 & .260 \\
\hline 08074800 & 795 & 1,050 & 1,220 & 1,430 & 1,580 & 1,740 & 2.9030 & .141 & .100 \\
\hline 08074850 & 1,050 & 1,330 & 1,520 & 1,750 & 1,930 & 2,100 & 3.0260 & .112 & .250 \\
\hline 08075000 & 11,800 & 16,800 & 20,500 & 25,500 & 29,500 & 33,700 & 4.0760 & .180 & .250 \\
\hline 08075400 & 1,930 & 2,770 & 3,380 & 4,220 & 4,880 & 5,590 & 3.2940 & .179 & .270 \\
\hline 08075500 & 4,320 & 6,650 & 8,430 & 10,950 & 13,030 & 15,300 & 3.6440 & .216 & .250 \\
\hline 08075550 & 480 & 595 & 665 & 750 & 810 & 870 & 2.6800 & .111 & .010 \\
\hline 08075650 & 2,110 & 3,060 & 3,730 & 4,620 & 5,310 & 6,020 & 3.3280 & .190 & .080 \\
\hline 08075730 & 2,170 & 2,920 & 3,420 & 4,050 & 4,530 & 5,000 & 3.3380 & .153 & .060 \\
\hline 08075760 & 510 & 695 & 820 & 990 & 1,120 & 1,250 & 2.7130 & .155 & .190 \\
\hline 08075770 & 1,370 & 2,120 & 2,690 & 3,510 & 4,180 & 4,910 & 3.1450 & .219 & .230 \\
\hline 08075780 & 305 & 480 & 615 & 810 & 975 & 1,150 & 2.4890 & .231 & .200 \\
\hline 08075900 & 1,680 & 2,640 & 3,390 & 4,470 & 5,360 & 6,350 & 3.2330 & .228 & .240 \\
\hline 08076000 & 3,070 & 4,590 & 5,720 & 7,310 & 8,600 & 9,980 & 3.5000 & .201 & .250 \\
\hline 08076200 & 630 & 840 & 990 & 1,190 & 1,340 & 1,500 & 2.7960 & .141 & .280 \\
\hline 08076500 & 2,060 & 2,770 & 3,260 & 3,930 & 4,450 & 5,000 & 3.3120 & .146 & .300 \\
\hline 08076700 & 5,760 & 8,710 & 10,900 & 14,000 & 16,600 & 19,300 & 3.7690 & .207 & .240 \\
\hline 08077100 & 255 & 325 & 370 & 430 & 470 & 515 & 2.4080 & .123 & .200 \\
\hline
\end{tabular}


analysis except that the expected value of the mean square error is used as an indicator of error instead of the regression variance. Application of this method produced flood-frequency values which were more heavily weighted toward the simulated data at lower recurrence intervals. This result was the opposite of those found on rural watersheds (Wibben, 1976; Thomas and Corley, 1977; Curtis, 1977; 01in and Bingham, 1977).

The method of averaging the results from the observed and simulated data for each recurrence intervals was used to produce the $Q_{t}$ values used as dependent variables (table 8 ). From a practical standpoint, however, there is little difference between the three weighting methods. Comparisons between the various weighted values of $Q_{t}$ showed that the values produced for $Q_{t}$ by the other methods were always within +21.5 and -16.8 percent of the $Q_{t}$ values obtained by the averaging method. More than 93 percent of the $Q_{t}$ values produced by the other methods were within \pm 10 percent of the values used.

Comparisons of the results of predictions based on equations developed from the various sets of weighted $Q_{t}$ values showed that regardless of the method of weighting used the predicted $Q_{t}$ values were within +10.9 and -8.7 percent of the final predicted $Q_{t}$ values. Only 1.3 percent of the $Q_{t}$ values predicted from equations based on the other weighting methods differed by more than \pm 7.5 percent from the final predicted values.

\section{DEVELOPMENT OF REGRESSION RELATIONSHIPS}

Multiple-regression techniques were used to define regional relationships to predict flood-peak magnitudes from drainage-basin characteristics. The dependent variables are the flood-frequency values in table 8 . The independent variables are given in table 5. The value of the variables were transformed to base 10 logarithms prior to performing the regression analysis.

Several regression models involving these independent variables were investigated. These included the following forms:

and

$$
\begin{aligned}
& \text { (a) } Q_{t}=a A^{b_{1} b^{b_{2}} A_{D} b_{3}} \\
& \text { (b) } Q_{t}=a A^{b_{1}} b^{b_{2}}
\end{aligned}
$$

$$
\text { (c) } Q_{t}=a A^{b_{1}}\left[K\left(1.0+0.01 A_{D}\right)\right]^{b_{2}}
$$

where $Q_{t}=$ discharge in cubic feet per second for a recurrence interval of $t$ years;

$A, K, A_{D}=$ the independent variables as defined previously;

$b_{1}, b_{2}, b_{3}=$ regression coefficients; and

$$
\mathrm{a}=\text { regression constant. }
$$

The $Q_{t}$ values predicted from these three forms were compared for the sites of this study. The difference between the various predicted $Q_{t}$ values was always within +8 percent and were within \pm 5 percent over 98 percent of the time. 
Table 8.--Flood-frequency characteristics used as dependent variables

\begin{tabular}{|c|c|c|c|c|c|c|c|}
\hline \multirow[t]{2}{*}{ Sequence } & \multirow[t]{2}{*}{ Station } & \multicolumn{6}{|c|}{$\begin{array}{l}\text { Flood-peak discharge } \\
\text { (cubic feet per second) }\end{array}$} \\
\hline & & $\mathrm{Q}_{2}$ & $Q_{5}$ & $Q_{10}$ & $Q_{25}$ & $Q_{50}$ & $Q_{100}$ \\
\hline 1 & 08074150 & 840 & 1,370 & 1,740 & 2,250 & 2,640 & 3,040 \\
\hline 2 & 08074200 & 235 & 350 & 420 & 505 & 565 & 625 \\
\hline 3 & 08074250 & 2,320 & 3,670 & 4,590 & 5,740 & 6,550 & 7,360 \\
\hline 4 & 08074500 & 7,400 & 11,340 & 14,140 & 17,850 & 20,760 & 23,780 \\
\hline 5 & 08074780 & 170 & 315 & 440 & 650 & 835 & 1,060 \\
\hline 6 & 08074800 & 745 & 1,040 & 1,220 & 1,460 & 1,640 & 1,810 \\
\hline 7 & 08074850 & 1,040 & 1,230 & 1,340 & 1,470 & 1,570 & 1,660 \\
\hline 8 & 08075000 & 12,160 & 18,020 & 22,260 & 27,960 & 32,460 & 37,150 \\
\hline 9 & 08075400 & 2,070 & 2,980 & 3,600 & 4,410 & 5,020 & 5,640 \\
\hline 10 & 08075500 & 4,340 & 6,940 & 8,850 & 11,460 & 13,540 & 15,720 \\
\hline 11 & 08075550 & 455 & 590 & 675 & 785 & 860 & 935 \\
\hline 12 & 08075650 & 1,830 & 2,980 & 3,840 & 5,080 & 6,080 & 7,150 \\
\hline 13 & 08075730 & 2,220 & 2,950 & 3,420 & 3,980 & 4,400 & 4,810 \\
\hline 14 & 08075760 & 475 & 645 & 760 & 905 & 1,010 & 1,120 \\
\hline 15 & 08075770 & 1,300 & 2,210 & 2,840 & 3,660 & 4,280 & 4,900 \\
\hline 16 & 08075780 & 315 & 470 & 575 & 720 & 830 & 950 \\
\hline 17 & 08075900 & 1,910 & 2,660 & 3,190 & 3,880 & 4,430 & 5,010 \\
\hline 18 & 08076000 & 3,210 & 4,800 & 5,960 & 7,560 & 8,840 & 10,190 \\
\hline 19 & 08076200 & 640 & 865 & 1,010 & 1,200 & 1,340 & 1,470 \\
\hline 20 & 08076500 & 2,060 & 2,740 & 3,200 & 3,780 & 4,210 & 4,660 \\
\hline 21 & 08076700 & 7,700 & 11,680 & 14,480 & 18,220 & 21,160 & 24,160 \\
\hline 22 & 08077100 & 250 & 340 & 400 & 470 & 520 & 570 \\
\hline
\end{tabular}


Form (a) was unsatisfactory because the variable AD did not remain statistically significant at the 5-percent significance level. Form (c) was formulated in an attempt not only to include the measure of urban development, $A_{D}$, but also to reduce the standard error of estimate resulting from form (b). Form (c) was developed by considering that the flood peak from an urbanized area in Houston was proportional to the product of bank-full channel conveyance, $K$, and a factor representing the amount of urban development. The factor, $1.0+0.01 \mathrm{AD}$, was selected on the basis of work done by Carter (1961). Because the standard error of estimate for form (c) was an improvement over that of form (b), form (c) was used for this study. Form (c) was evaluated for bias at the 5- and 50-year recurrence intervals. None was apparent.

The values determined for the regression constant and the regression coefficients are given in table 9. Station 08074780, Keegans Bayou at Keegans Road, was not used in the development of these relationships because of missing data. The variation of the constant and coefficients indicate consistency and continuity with the regression model. Table 9 also presents the standard error of estimate (Se) and the multiple-correlation coefficent (MCC) for the regional relationship at each recurrence interval.

The results of applying the relationships in table 9 to the stations used for this study are given in table 10. The flood-frequency values in table 10 should be considered as good estimates as long as conditions in the basin remain similar to those of December 1976. The effect of changes in a basin may be predicted by use of the relationships given in table 9.

\section{APPLICATION OF REGRESSION RELATIONSHIPS}

The regression relationships are shown in table 9. They provide a method for computing flood-peak magnitudes for recurrence intervals of $2,5,10,25$, 50,100 , and 500 years on ungaged and unregulated streams in the Houston, Texas, metropolitan area. These sites may require flood-frequency information for a variety of reasons. For example, the site may be a completely urbanized basin for which information is required to establish flood insurance rates; or the site may be completely undeveloped, but information is required by developers to determine predicted future flooding. In the first case, selection of the basin characteristics may be fairly straightforward, however, the second case can illustrate several possible errors in selecting basin characteristics. This section provides guidelines for computing the basin characteristics.

\section{Drainage Area}

The drainage area should be delineated on a U.S. Geological Survey 7-1/2minute or 15-minute topographic map, with the aid of field reconnaissance. In addition, the drainage basin should be inspected so that drainage ditches, which are not shown on the map, but which may cause variations in the total contributing drainage area, can be located. The field inspection may also discover other features, such as detention storage and storm sewers that will affect the total contributing drainage area. 
Table 9.--Regionalized relationships for $Q_{t}$

\begin{tabular}{rrrccr}
\hline $\begin{array}{c}t \\
\text { (years) }\end{array}$ & $\begin{array}{c}\text { Regression } \\
\text { constant }\end{array}$ & $\begin{array}{c}\text { Regression } \\
\text { coefficient } \\
\text { for } \\
\mathrm{A}\end{array}$ & $\begin{array}{c}\text { Regression } \\
\text { coefficient } \\
\text { for } \\
\text { K(1.0+0.01AD) }\end{array}$ & $\begin{array}{c}\mathrm{S}_{\mathrm{e}} \\
\text { (percent) }\end{array}$ & MCC \\
\hline 2 & 2.028 & 0.383 & 0.447 & 25.1 & 0.978 \\
5 & 2.208 & .392 & .468 & 19.7 & .987 \\
10 & 2.301 & .399 & .478 & 18.1 & .989 \\
25 & 2.460 & .410 & .487 & 17.1 & .991 \\
50 & 2.576 & .419 & .492 & 16.9 & .991 \\
100 & 2.710 & .428 & .495 & 17.1 & .991 \\
500 & 3.097 & .451 & .498 & 18.1 & .991 \\
\hline
\end{tabular}

Example: $\quad Q_{2}=2.028 \mathrm{~A}^{0.383}\left[\mathrm{~K}\left(1.0+0.01 \mathrm{~A}_{\mathrm{D}}\right)\right]^{0.447}$ 
Table 10.--Predicted flood-peak discharges for recurrence intervals of $2,5,10,25,50$, and 100 years

\begin{tabular}{|c|c|c|c|c|c|c|c|}
\hline \multirow{2}{*}{ Sequence } & \multirow[t]{2}{*}{ Station } & \multicolumn{6}{|c|}{$\begin{array}{l}\text { Flood-peak discharge } \\
\text { (cubic feet per second) }\end{array}$} \\
\hline & & $\mathrm{Q}_{2}$ & $\mathrm{Q}_{5}$ & $Q_{10}$ & $Q_{25}$ & $Q_{50}$ & $Q_{100}$ \\
\hline 1 & 08074150 & 1,230 & 1,780 & 2,130 & 2,610 & 2,970 & 3,310 \\
\hline 2 & 08074200 & 300 & 415 & 480 & 570 & 635 & 695 \\
\hline 3 & 08074250 & 1,660 & 2,420 & 2,920 & 3,600 & 4,120 & 4,600 \\
\hline 4 & 08074500 & 8,300 & 12,830 & 15,990 & 20,510 & 24,070 & 27,550 \\
\hline 5 & 08074780 & 565 & 790 & 930 & 1,120 & 1,260 & 1,400 \\
\hline 6 & 08074800 & 850 & 1,200 & 1,430 & 1,740 & 1,970 & 2,190 \\
\hline 7 & 08074850 & 740 & 1,050 & 1,240 & 1,500 & 1,690 & 1,870 \\
\hline 8 & 08075000 & 10,740 & 16,800 & 21,060 & 27,160 & 31,970 & 36,670 \\
\hline 9 & 08075400 & 2,210 & 3,250 & 3,940 & 4,900 & 5,630 & 6,330 \\
\hline 10 & 08075500 & 4,620 & 6,970 & 8,580 & 10,860 & 12,650 & 14,400 \\
\hline 11 & 08075550 & 420 & 585 & 685 & 815 & 910 & 1,000 \\
\hline 12 & 08075650 & 2,180 & 3,230 & 3,920 & 4,860 & 5,560 & 6,220 \\
\hline 13 & 08075730 & 1,420 & 2,060 & 2,470 & 3,040 & 3,460 & 3,850 \\
\hline 14 & 08075760 & 515 & 725 & 850 & 1,020 & 1,140 & 1,260 \\
\hline 15 & 08075770 & 2,050 & 3,010 & 3,650 & 4,530 & 5,190 & 5,820 \\
\hline 16 & 08075780 & 370 & 500 & 585 & 700 & 785 & 865 \\
\hline 17 & 08075900 & 1,500 & 2,160 & 2,600 & 3,210 & 3,680 & 4,140 \\
\hline 18 & 08076000 & 3,350 & 4,970 & 6,080 & 7,650 & 8,880 & 10,090 \\
\hline 19 & 08076200 & 570 & 795 & 935 & 1,130 & 1,270 & 1,410 \\
\hline 20 & 08076500 & 1,610 & 2,320 & 2,790 & 3,460 & 3,960 & 4,450 \\
\hline 21 & 08076700 & 8,560 & 13,170 & 16,400 & $21,1.10$ & 24,870 & 28,610 \\
\hline 22 & 08077100 & 255 & 345 & 400 & 475 & 525 & 570 \\
\hline
\end{tabular}


The term "controlling reach" as used in this report refers to that reach of a channel, downstream from the site in question, in which the frictional resistance of the streambed and banks determine the rate of flow at a given stage. Because flat slopes and relatively slow water velocities are characteristic of streams in the Houston area, reach control is typical except at extremely low stages. Reach control must exist at bank-full stage if the technique described in this report is to be applied. Before determining bankfull channel conveyance, then, the reach downstream from the site should be inspected to insure that there are no dams, culverts, or other physical features that could cause an appreciable break in the water-surface slope.

The regional relationships shown in table 9 may be used as aids in the design of channels to carry future flood waters. When the relationships are used for this purpose, some guidelines are required with respect to the magnitude of channel conveyance. A relationship of maximum-allowable channel conveyance (KMAX) to drainage area as shown on figure 2 was determined by plotting the values for $K$ and $A$ for all sites used in the study. The assumption was made that none of the channels were adequately designed to carry the maximum discharges likely to occur when the basins are completely urbanized.

Figure 2 was developed by using a straight-line relationship through points 5 percent greater than those having the greatest ratio of $K$ to $A$. The user should be aware that KMAX is not intended to be an optimal value. It should be considered a boundary for which the value of $K$ substituted into the relationships developed by this study cannot exceed. If the computed $K$ is greater than KMAX, then KMAX should be used. However, if the computed $K$ is less than or equal to KMAX, the assumption should not be made that the designed channel conveyance is acceptable for future development without considering other economic and engineering variables.

Bank-full channel conveyance may be determined at existing sites as follows: (1) Obtain two or more representative cross sections of the channel in the controlling reach; (2) select the elevation of the top of the lowest bank as bank-full stage for each cross section; (3) select Manning's $n$ and compute the bank-full conveyance, $K$, for each cross section; and (4) determine the arithmetic mean value of $K$ for the site.

\section{Percentage of Urban Development}

The percentage of urban development, $A_{D}$, may be determined by the use of aerial photographs. After delineating the total contributing drainage area on the photographs, the 200-foot boundaries for urbanized areas within the drainage basin may be easily marked either on the photographs or on a transparent overlay. If aerial photographs are not available, field reconnaissance will yield current conditions. The developed area as delineated may be measured in square miles by planimetering. This value is then converted to a percentage of the total contributing drainage area.

In summary, the relationships are applied at ungaged and unregulated sites in the Houston, Texas, metropolitan area by the following procedure: (1) Locate the site on a map; (2) determine the total contributing drainage 


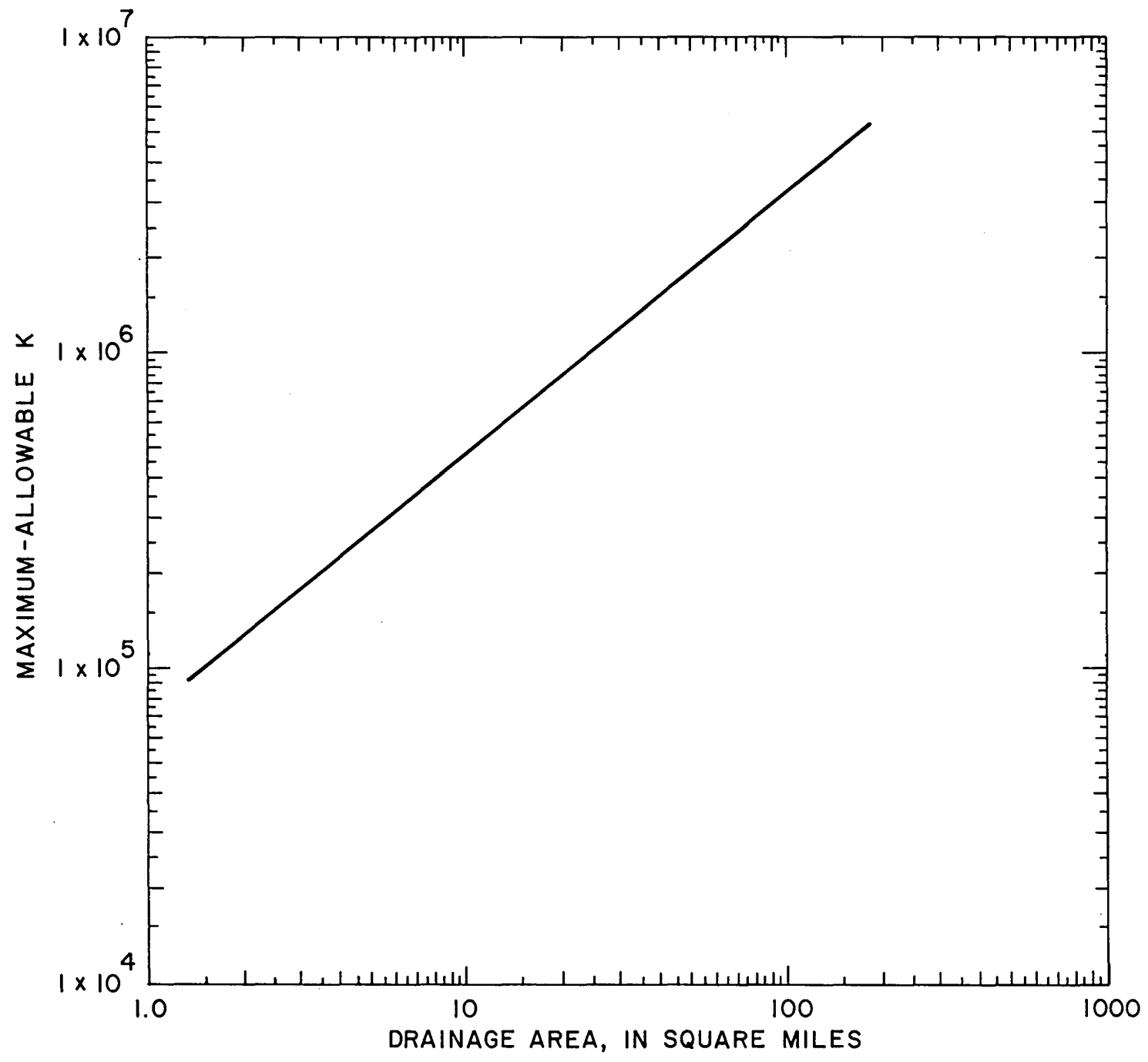

FIGURE 2.-Maximum allowable conveyance versus drainage area 
area; (3) define the urbanized area for the date required; (4) compute bankfull channel conveyance for the controlling reach of the channel downstream from the site; and (5) compute flood-frequency values by the use of the regression equations in table 9 .

The technique may also be used to evaluate the effects of future development. Thus, various stages of urbanization may be assumed to predict urban flood-frquency characteristics at these different stages of development.

\section{Limitations and Special Cases}

The technique presented in this report does not apply to regulated streams. Flood-frequency estimates are defined for unregulated streams in the Houston area within the following limits of basin characteristics.
(1) Contributing drainage area, A
1.33 to 182 square miles
(2) Bank-full channel conveyance, $K$
(3) Percentage of urban development, $A_{D}$
$1.2 \times 10^{4}$ to $2.8 \times 10^{6}$
37.0 to 98.9

The following are some special cases in which the technique may be used: (1) Use of the technique to predict changes due to urbanization; (2) use of the technique at a site where bank-full channel conveyance has an abrupt change; and (3) use of the technique in a design situation. These are discussed below.

The use of the technique to predict changes due to urbanization is easily accomplished if the user wants to compare current conditions with conditions of complete urbanization. The percentage of urban development, $A_{D}$, is increased to 100 and $K_{\text {MAX }}$ is determined from figure 2. The flood frequency estimates obtained by using these values with the relationships in table 9 will indicate the effects of complete urbanization and complete rectification of a channel.

If the user wants to compare a site in its completely rural state to a completely urbanized state, some assumptions must be made. Values for $A D$ at several rural sites in the Houston area were computed at between 15 and 25 percent. Thus, AD for rural sites is chosen as 20 percent. Assuming that KMAX may be selected from figure 2, the bank-full channel conveyance for a completely unrectified (rural) channel was chosen by assuming that Halls Bayou at Deertrail Street and Greens Bayou at U.S. Highway 75 were typical of such streams, for which the average ratio of KMAX to KRURAL equals 15. This value is assumed to be near the upper limit; therefore, the effects of urbanization on flood-frequency characteristics should not be greater than those given in table 11. At any site in the Houston area, a change from completely rural to completely urban conditions should not increase the peak discharge of a 100-year flood by more than a factor of 4.9 .

An abrupt change in channel conveyance may occur at a site where channel rectification has halted. Use of the technique at such a site requires the computation of flood-frequency characteristics for both the larger and smaller values of $K$. The flood-frequency characteristics computed by using the smaller $K$ would be valid for the period that the abrupt change in the channel remains. Once the channel rectification is complete, the flood- 
Table 11.--Effects of urbanization on

flood-frequency characteristics

\begin{tabular}{|c|c|c|c|c|c|c|c|}
\hline \multirow{3}{*}{$\begin{array}{r}\text { Case } \\
\text { no. }\end{array}$} & \multirow{3}{*}{$\begin{array}{l}\text { Description } \\
\text { of change } \\
\text { due to } \\
\text { urbanization }\end{array}$} & \multicolumn{6}{|c|}{$\begin{array}{l}\text { Factor by which } \\
\text { flood peak increases }\end{array}$} \\
\hline & & & ecurr & ice is & terva & (yea & \\
\hline & & 2 & 5 & 10 & 25 & 50 & 100 \\
\hline 1 & $\begin{array}{l}\text { Development increases from } \\
\text { completely rural to com- } \\
\text { pletely urban }\end{array}$ & 1.26 & 1.27 & 1.28 & 1.28 & 1.29 & 1.29 \\
\hline 2 & $\begin{array}{l}\text { Channel is changed from com- } \\
\text { pletely unrectified (rural) } \\
\text { to completely rectified state }\end{array}$ & 3.36 & 3.55 & 3.65 & 3.74 & 3.79 & 3.82 \\
\hline 3 & $\begin{array}{l}\text { Cases } 1 \text { and } 2 \text { occur } \\
\text { together }\end{array}$ & 4.23 & 4.51 & 4.67 & 4.78 & 4.89 & 4.93 \\
\hline
\end{tabular}


frequency characteristics computed by using the larger $K$ would be valid for such a site, assuming the larger $K$ did not exceed $K_{\text {MAX }} \cdot$

If the technique is being used to estimate $Q_{t}$ values for a channel design, the value of $K$ used for the planned channel should not produce unreasonably large estimates of $Q_{t}$. The following procedure is suggested to check the value of K: (1) From the design configuration of the planned channel, compute K; (2) determine $A$ for the basin; and (3) enter figure 2 with the value of $A$ and determine $K_{\text {MAX. If }} K$ is greater than $K_{\text {MAX }}$, then KMAX must be used in determining the $Q_{t}$ values. If $K$ is equal to or less than KMAX, the computed $K$ must be used in these relationships. Whether or not the designed channel should be built may be determined by other considerations.

The user of the equations in table 9 is cautioned that the use of a $K$ value larger than $K_{\text {MAX }}$ may result in the computation of peak discharges larger than the basin can produce. This problem should be avoided by limiting the maximum value of $K$ substituted into these relationships to $K_{\operatorname{MAX}}$ (fig. 2). Also, the user should never confuse the bank-full channel conveyance value computed for this technique with the actual channel conveyance required by various flows. 
Carter, R. W., 1961, Magnitude and frequency of floods in suburban areas: U.S. Geological Survey Professional Paper 424-B, p. B9-B11.

Curtis, G. W., 1977 [1978], Frequency analysis of Illinois floods using observed and synthetic streamflow records: U.S. Geological Survey WaterResources Investigations 77-104, $38 \mathrm{p}$.

Espey, W. H., Jr., and Winslow, D. E., 1968, The effects of urbanization on unit hydrographs for small watersheds, Houston, Texas, 1964-67: Tracor Document No. 68-975-U, submitted to the Office of Water Resources Research, pt. 1, 70 p.

Hardison, C. H., 1971, Prediction error of regression estimates of streamflow characteristics at ungaged sites: U.S. Geological Survey Professional Paper 750-C, p. C228-C236.

Johnson, S. L., and Sayre, D. M., 1973, Effects of urbanization on floods in the Houston, Texas metropolitan area: U.S. Geological Survey WaterResources Investigations $3-73,50 \mathrm{p}$.

0lin, D. A., and Bingham, R. H., 1977, Flood frequency of small streams in Alabama: Alabama Highway Department HPR No. 83, 44 p.

0stle, B., 1966, Statistics in research: Ames, The Iowa State University Press, $585 \mathrm{p}$.

Thomas, W. 0., Jr., and Corley, R. K., 1977, Techniques for estimating flood discharges for Ok lahoma streams: U.S. Geological Survey Water-Resources Investigations $77-54,180 \mathrm{p}$.

U.S. Water Resources Council, 1977, Guidelines for determining flood flow frequency: Hydrology Committee Bulletin 17A, 26 p., 14 apps.

U.S. Heather Bureau, 1961, Rainfall frequency atlas of the United States: U.S. Weather Bureau Technical Paper No. 40, 109 p.

Wibben, H. C., 1976 [1977], Application of the U.S. Geological Survey rainfallrunoff simulation model to improve flood-frequency estimates on small Tennessee streams: U.S. Geological Survey Water-Resources Investigations $76-120,59 \mathrm{p}$. 